routine can be used to assess sequential dependencies between successive IRTs and to relate response durations to the IRTs that precede them. Another F3 of this sort records all possible combinations of two events in a sequence of six occurrences of either event.

One SKED user (Frank Butler, personal communication, 1973) has developed a set of mathematical operations that can be applied to record counter values. This set of subroutines even permits the calculation of means and standard deviations on-line through the use of these operators. Unfortunately, this package was developed for a rather specific and unusual configuration of hardware, but the packages could be easily rewritten for use on most machines with only a minimum of effort.

\section{LOADING F3s}

Since the loader in the RTS tries to enter state tables into core unoccupied by the RTS monitor, it is necessary to protect any F3 subroutine from being overwritten by state tables. There are two methods for loading F3s. The first of these is to request space for the F3 by means of the dialogue when establishing the system. This serves well for F3s located in Field 0. For longer F3s, or for F3s that are to be used only infrequently, it is often more convenient to load the assembled F3 on top of the RTS. The required protection can be obtained by storing the $\mathrm{F} 3$ at the end of one of thefields of memory and by then adjusting one parameter in the RTS to protect this area.

Documentation of some subroutines within SKED, as well as of some parameters of the current state table that will be of use to programmers, has been prepared. Some differences exist between the $4 \mathrm{~K}$ and the multiple field systems that must be taken into account when writing new F3s. A library and documentation of F3s has been prepared and is available from the SKED users' group.

\title{
FOCAL, FORTRAN, and BASIC programs for reformatting and analyzing data collected by the SKED program*
}

\author{
ARTHUR SNAPPER, DENNIS LEE, LEONARD BURCZYK, and JOSE C. SIMOES-FONTES \\ Western Michigan University, Kalamazoo, Michigan 49001
}

\begin{abstract}
Several programs have been written in the FOCAL, FORTRAN, and BASIC languages for reformatting and analyzing SKED data. These programs include selection and explicit labeling of sets of recording counters representing distributions and/or total counts of events, several general manipulations of distributional data, and standard statistical treatment of distributions.
\end{abstract}

In the behavioral sciences, the amount and complexity of data analysis varies widely, even within the same laboratory. Some experimental questions are amenable to simple comparisons of response rates. Other questions deal with the microanalysis of the behavior stream and require extensive manipulations of data. Most research falls between these extremes.

SKED was designed primarily for the purpose of data acquisition and experimental control. However, some simpler forms of data reduction are easily notated and programmed with the SKED software. The addition of software subroutines, of course, can provide sophisticated data treatment on-line. Limitations on the available amount of core storage and limitations on the time within which the system must respond to new inputs are the two major parameters of an on-line system. Limited core storage restricts the quantity of stored data and thus limits the amount of data that can be acquired. Temporal limitations, on the other hand,

\footnotetext{
* This work was supported in part by Research Scientist Development Award K2-MH-70483 from the National Institute of Mental Health to the senior author.
}

reduce the amount of mathematical operations that can be performed on data on-line if the system is to respond to inputs quickly enough. Otherwise, summary statistics might be obtained during the data collection period so that large quantities of data need not be retained in the computer. A balance between these two restraints is achieved by performing simple sorting and summing operations while acquiring data. More extensive data treatment is then left to further analysis off-line, either on the same computer or on a second system.

One other commonly used technique to obtain raw data without forcing it into a prespecified data reduction format is to store new data on an external medium such as magnetic tape or disk, so that minimal core is required for storage, and data analyses is not accomplished during the acquisition period. Even with this system, it is usually desirable to accumulate some minimal amount of data in core before recording it on an external device, since such devices are most efficient when they have fixed-length formats that demand a certain number of characters on each data transfer. Of course, with this scheme, it is necessary to process the data off-line by the 
same or by a different computer that can read the stored data.

Only with the simplest sorts of data treatment will the daily recording be in final form. Even when manipulation of the daily data that has been arquired on-line is not extensive, it is often desirable to fverage data across sessions or Ss and to use the computer to plot each day's data on the same graph that contains earlier data. The present account describes some general data analysis packages that have been written to handle SKED data. These programs are written in FOCAL, FORTRAN, and BASIC, which are commonly used mathematical computing languages that permit powerful manipulation of data.

FOCAL is the simplest of these languages and can be used in a $4 \mathrm{~K}$ PDP-8, although core limitations on the complexity of the program will of ten demand at least $8 \mathrm{~K}$. FOCAL is paper tape oriented and is not as powerful as the other languages. However, for the user with only paper tape or magnetic cartridge for data storage, FOCAL may be the optimal language.

BASIC is also a simple language to use, but it is more powerful than FOCAL. It includes commands that can deal with more powerful file-oriented peripherals such as magnetic tape or disk. BASIC might well be the language of choice for a user with the PDP-8 OS 8 system and intermediate analysis requirements.

FORTRAN is the most powerful of these languages. It has been used for a long time, and there is an extensive library of routines to perform almost every function. FORTRAN is available in different versions or levels, which are basically compatible, but there might be some differences in the versions of FORTRAN available on different computers. OS 8 supports both FORTRAN II and FORTRAN IV. The FORTRAN IV language for PDP-10 is used for the programs presented here, but some version of FORTRAN will be the choice of users requiring extensive data analysis.

Before describing these routines in greater detail, it is worth characterizing the sort of data reduction achieved by SKED on-line.

\section{Total Responses in Different Temporal or Stimulus Conditions}

The simplest type of data collection and treatment is the collection of the total number of responses under various conditions in separate recording counters. For example, responses in successive 5-min periods of a session might be stored in separate counters to assess temporal changes in rate throughout the session. Counter 1 ( $\mathrm{C} 1$ ) might then represent the total number of responses in the first $5 \mathrm{~min}, \mathrm{C} 2$ would contain the count in the second 5, etc. Actual calculations of response rate (i.e., responses per minute) would then be deferred for off-line processing. An example of total response count under different stimulus conditions might be a count of the number of responses in each component of a multiple schedule. Cl would then represent responses in
Stimulus Condition 1 and $\mathrm{C} 2$ would contain those in Stimulus Condition 2. Again, actual rates would be calculated off-line.

\section{Frequency Distributions}

The next level of complexity in data acquisition schemes is the sorting of data into frequency distributions. One example would be to categorize the time between responses into successive intervals and to tally the number of interresponse times (IRTs) falling into each category. Further analysis of the IRT distribution to be conducted off-line would include calculating relative frequencies, conditional probabilities (IRT/OP), means, and variances.

A related distribution commonly obtained is the distribution of response counts in successive portions of a fixed interval. In this case, the interval might be divided into 10 equal parts, and the number of responses in each tallied in separate counters. Off-line calculations might include relative frequencies, rates, and measures of temporal discriminations, such as quarter-life and curvature index.

A third simple type of frequency distribution is a latency distribution of the time from the onset of a stimulus until a response is emitted. An IRT distribution and a postreinforcement-pause distribution are also distributions of this type. Off-line calculations would be of the same sort applied to IRT distributions already discussed.

A fourth distributional measure is the collection of latencies between successive ordinal responses in a fixed ratio. For example, the total time between the first and second response in the ratio might be collected in $\mathrm{C} 1$, the time between the second and third in $\mathrm{C} 2$, etc. Once again, off-line analysis would be similar to analysis of the subinterval distribution.

In all of the above cases, several alternatives are possible. Units of the distribution need not be equal, for example. It might be interesting to collect IRTs in log units of time since the preceding response.

More than one distribution might be taken from the same $S$ in the same session. For example, the time course of changes in IRTs might be cbtained, or separate IRT distributions might be obtained in different components of a multiple schedule.

\section{Distributional Matrices}

One type of more complex analysis that is easily obtained on-line by means of an F3 machine language subroutine is a matrix of response tallies in which the rows of the matrix represent values of a second distributional variable. This arrangement permits obtaining one distribution as a function of a second. For example, a correlation between the length of an IRT and the length of the preceding IRT might be obtained. Another example would be the duration of a response as a function of the IRT it terminates. In both of these 
cases, both variables would be specified in distributional format.

\section{Format of SKED Data}

SKED data format may be seen in the following example:

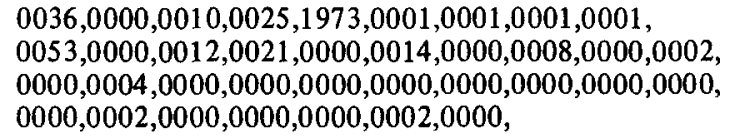

The first line of numbers is the identification code. Number 1, the first four-digit number contains a value equal to the number of recording counters in the printout plus the nine identification numbers. The second number contains the number of the station or box that generated the data. The third, fourth, and fifth numbers of the first line contain the month, day, and year, which are entered by Teletype before the session. The sixth, seventh, and eighth numbers identify the experiment, $S$, and group, and they are established when the state table is loaded under control of the run-time system (RTS). The ninth number contains the recording number, which is incremented each time data is recorded for this station. The value of this number will be 1 for the first recording within a session, 2 for the second, etc. Following the first line of numbers are the data expressed as successive four-digit decimal numbers with commas terminating each number. A maximum of 10 counters per line are printed. The contents of each counter are specified by the state table of the particular experiment and the behavior of the $\mathrm{S}$.

Each recording counter has a maximum value of 4096, but there is a method for reserving the next counter to record overflow. Each count in an overflow counter represents 4,096 counts in the preceding counter, which is reset to zero when overflow occurs.

Since more than one distribution can be contained in a recording of a session, a useful feature of any data analysis routine is the ability to select a set of counters from the recording, excluding all other counters, to produce a new record containing only the distribution of interest.

An example of a state program, the data collected by it, and analysis of that data by one of the FOCAL programs follows:

/IRT DISTRIBUTION GENERATOR

/ON1=SESSION LIGHT

/ON2=REINFORCEMENT

/R1=RESPONSE LEVER

/C1-C2=TOTAL RESPONSES WITH OVERFLOW IN C2

/C3=REINFORCED RESPONSES

/C4-C27=IRT's IN 1" BINS WITH OVERFLOW

/DRL5"

/REQUEST 27 COUNTERS

S.S.1,

S1,

R2:ON1:F2(A,4)-S2/START EXPERIMENT WITH TTY RESPONSE
S2,

$1^{\prime \prime}: \mathrm{Z} 1 ; \mathrm{F} 1(\mathrm{~A}, 2,27)-\mathrm{S} 2 /$ INCREMENT BIN NO. $\mathrm{R} 1: \mathrm{C} 1{ }^{*} ; \mathrm{F} 2(\mathrm{~A}, 4)-\mathrm{S} 2 / \mathrm{RECORD}$ AND RESET TIMER Z2-S3/WAIT FOR END OF REINFORCEMENT

S3

Z3-S2/RESTART TRIAL AT END OF REINFORCEM

S.S.2,

S1,

R1-S1/RESET COUNT FOR PREMATURE R1. 5Z1-S2/REINFORCEMENT SET-UP AFTER 5"

S2,

R1:ON2;C3;Z3--S3/REINFORCE

2":OFF2;F2(A,4);Z3--S1/END REINFORCEMENT

$\$$

$0036,0000,0010,0025,1973,0001,0001,0001,0001$, $0053,0000,0012,0021,0000,0014,0000,0008,0000,0002$, $0000,0004,0000,0000,0000,0000,0000,0000,0000,0000$, $0000,0002,0000,0000,0000,0002,0000$,

FREQ

BOX \#: $\emptyset$

DATE: $10: 25: 1973$

EXP. \#: 1

SUB \#: 1

GROUP \#: 1

REC. \#: 1

BIN SIZE $=: 1$

1ST CTR $=: 4$

LAST $=: 27$

OVERFLOW: YES

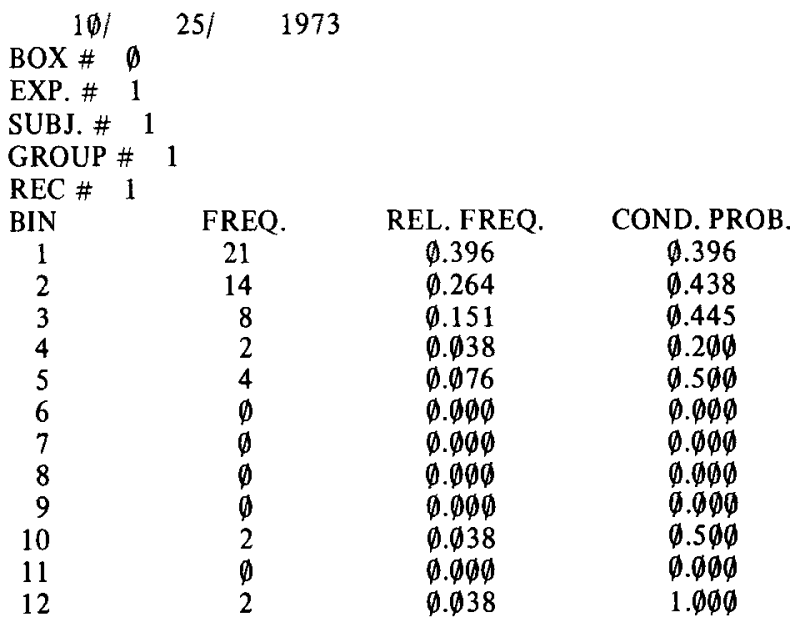

TOTAL FREQ. $=53$

$\operatorname{MEAN}=\emptyset .278846 \mathrm{E}+\emptyset 1$

VAR. $=\emptyset .136125 \mathrm{E}=\emptyset 2$

The state table is the program for a reinforcement schedule called DRL (differential reinforcement of low rates), in which responses must follow preceding responses or the end of the preceding reinforcement by at least $5 \mathrm{sec}$ for a reinforcement to be delivered. Responses occurring before the 5-sec delay period has elapsed are not reinforced but start a new 5-sec delay. This schedule typically results in the S's forming a good temporal discrimination with most interresponse times falling near $5 \mathrm{sec}$. State Set 1 (S.S.1) starts in State 1 (S1). An R2 response generated by the $\mathrm{E}$ initiates the 
session and turns on the houselight. R2 also sets an IRT distribution to Recording Counter 4 (the first recording counter in the distribution). State 2 of S.S.1 is both a recording and a timing state. Every $1 \mathrm{sec}$ following a response, the recording counter fdesignated hy the variable $A$, which determines the counter in which responses will be recorded) is incremented by 2. 1. very response is recorded in the counter selected by the value of A when the response occurs, after which the response resets $\mathrm{A}$ to 4 for the beginning of the next IRT measurement. Every leverpress, reinforced or not, is also recorded in Counter 1 , the response counter for the session response rate. Thus, if the time between the first response and the next response is less than $1 \mathrm{sec}$, the response will be recorded in Counter 4 . With a 1-2-sec delay, the response will be recorded in Counter 6 , etc. Counters $5,7,9$, etc., are overflow counters that will be incremented by 1 when a count of 4096 occurs in the preceding counter. These are reserved by the asterisk $\left({ }^{*}\right)$ following the variable, A.

State Set 2 controls the delivery of reinforcement. The completion of five $\mathrm{Zl}$ pulses $(5 \mathrm{sec})$ sets up reinforcement for the next response, unless a premature response resets the accumulated count. In the example, a recording is shown followed by a data analysis of the IRT distribution produced by a program written in the FOCAL language (a similar program has been written in BASIC). The frogram begi by asking thie operator to identify the record to be analyzed by specifying the identification code of the first line of the record, including the Box \#, the date, the Exp., Sub., Group, and Recording \#s. The responses of the operator are italicized, and all other text is produced by the program. Next, the operator is asked to specify the bin size of the IRT distribution (the program assumes constant bin width for all bins), the first counter of the distribution, the last counter of the distribution, and whether there are overflow counters included in the distribution. After the last response, the computer checks the file for the record with the correct identification code. If the first recording has the correct code, the computer analyzes the data and prints the bin number, absolute frequency of counts in each bin, the relative frequency, and the conditional probability or IRT/OP analysis. Finally, the computer prints the total frequency of the distribution, the mean of the distribution, and its variance. If the first recording does not have the correct identification, the program then searches the file for a recording with the specified code.

\section{FORTRAN AND BASIC PROGRAMS}

The FOCAL program expects to read files that include recordings of the specified identification code. Other recordings may be on the same paper tape, but they will be ignored. Only one specific recording can be analyzed at one time. This fact is due to the limitations of FOCAL and available core in which it operates. The latter limitation restricts the length of the program and the number of data values that can be stored in the computer. Other analysis of the same data must be accomplished alter the first analysis has been completed.

For users possessing magnetic tape or a disk, it is possible to use the external mass storage device to run more extensive programs with the more powerful BASIC and FORTRAN programs. A version of a FORTRAN analysis program has been developed on a PDP-10 computer, a large time-sharing system. The discussion of this program and the considerations that led to its development will have direct implications in terms of program format, dialogue, and analysis subroutines for users with minisystems. However, the program will have to be modified to run under a smaller system.

The first consideration is the transfer of files from the smaller RTS to the larger off-line analysis system. This can be accomplished with PIP (peripheral interchange program) and low-or high-speed paper tape, although this method is slow when large quantities of data must be transferred each day. The PIP instruction for the transfer requires the specification of a file name for the data to be stored on disk at the time of the transfer. For later convenience, the numerical date on the file is used as the name for all of the recordings collected on any one day. For example, a session of data collected October 31, 1973, would be transferred to the disk from the Teletype reader with the following instruction: DSK : 103173.DAT $\leftarrow$ TTY:. The first six characters of the file name contain the date 10/31/73. The extension name. DAT is required for data files.

Even the larger time-sharing computers have limitations on the amount of storage available to the user at any one time. The quantity of data that it is possible to amass with a medium-to-large RTS system can be considerable. It is our experience that as many as 100,000 recording counters is not unreasonable for a laboratory running 10 stations a day on 10 experiments. Some experiments, in fact, can require that many counters by themselves (e.g., interbeat times in a heart-rate conditioning study). Since it is often desirable to average or compare data across sessions or days, it is necessary to retain data for at least 1 month in a form that is relatively accessible to the computer for such cross-comparisons.

Fortunately, larger systems have solutions to problems of this sort, possessing mass storage peripherals, including disk files and magnetic tape. We handle the storage problem by using the PIP commands to transfer each day's file to magnetic tape as well as to retain it briefly on disk for initial analysis. If a comparison is to be made across sessions, then the stored files can be recalled from magnetic tape to the disk by means of the file name which was coded by calendar day. When the appropriate data has been entered into the disk from paper tape or disk, the main analysis can take place.

Several different data analysis routines, both specific 
and general, have been programmed in the FORTRAN language. However, rather than write each routine as a stand-alone version, as is necessary in FOCAL, we decided to incorporate these routines in a larger package which shares several common features necessary for each routine.

\section{OPTION Command}

The first command of the data analysis package is the option command, which permits the operator to select from a library of routines, each of which performs a different function on the data. The user types OPTION and the number representing the particular analysis that he desires. For example:

\section{OPTION \# $\quad$ SPECIFIC ANALYSIS}

1 Total count in specified counters

2 Average count in specified counters

3 Rate (count/bin size)

4 Bin, frequency, relative frequency

5 Bin, frequency, relative frequency, conditional probability

6 Mean and variance of a distribution

7 Quarter life and curvature index of a distribution

8 Divide specified distribution by second distribution

9 Open for new routines

10 Graph a particular distribution

These analysis options are contained in the computer in subroutine formats. The program permits additional subroutines to be added when new analysis programs have been written.

\section{SEARCH Routine}

The search command is the next instruction to the data analysis routine. This command has the function of examining the daily files that may contain data recordings from more than one experiment, $S$ group, and recording number. From the daily files, the search routine will extract the recordings specified by parameters of the search command and create a new, smaller file, containing only the recordings to be used in the particular analysis specified in the option command. The search routine can obtain recordings from more than one daily file if the daily files are stored in the currently accessible mass-storage device. The following illustrates the use of the search command:

Search 101573.DAT;1;1,2;ALL;0;9.

File 101573.DAT will be examined for recording \#9 of Box 0 for all Ss who belong to Groups 1 and 2 of Experiment 1. An example of a search command that obtains data from two files is:

\section{Search 061972.DAT,071972.DAT;3,1;36;5;115.}

Files representing sessions on 6/19/72 and $7 / 19 / 72$ will be searched for Recordings 1 through ( $\uparrow) 5$ of Box 5, in which S 36 of Group 1, Experiment 3, was run. This allows the production of a smaller file to be produced in which the relevant data is contained.

\section{FOR Command}

After preliminary sorting has been accomplished by SEARCH, the specific counters are further delineated by the FOR command. With this command, the counters to be analyzed are specified along with the overflow option, and the bin size or other parameter of the option currently being used. To obtain Counters $3-7$ with a bin size of $5 \mathrm{sec}$ and no overflow, the FOR command would be:

$$
\text { FOR } 5 ; 3 \uparrow 7 ; 0 \text {. }
$$

\section{Output}

Output of the analysis is automatic for each option, but it may be directed within options to the Teletype, line-printer, or mass-storage file for further processing. Some users may want to store a reduced and summarized version of the daily data to conserve total storage space. One additional command prints the specific recording or recordings on the Teletype for inspection and storage as hard copy. The command is called PRINT, and it has the same arguments as the search command.

The FOCAL system described and its BASIC counterpart are available for small systems. The FORTRAN subroutines and the commands OPTION, SEARCH, and FOR are subject to modification for adaptation to the OS 8 , but are operational as described for large time-sharing systems. 